\title{
Mesure des poussées hydrauliques des turbines Francis à axe vertical
}

\section{The measurement of hydraulic thrust on a vertical Francis turbine}

\author{
PAR M. S. X. CASACCI

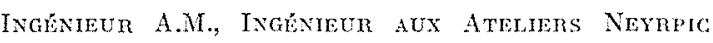 \\ $\mathrm{ET}$ \\ M. P. JARRIAND

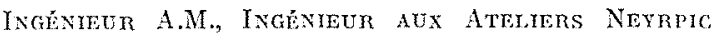

English synopsis p. 311

Pour déterminer les dimensions et les caractéristiques de fonctionnement d'une pivoterie, il est nécessaire de comnaîlue exactement la charge qu'elle supporte. Celle-ci, dans le cas de turbines hydrauliques à axe vertical, comprend le poids des pièces tournantes et la poussée sur la roue qui, pour certaines installations, constitue la majeure partie de la charge.

Le calcul ne permet pas la détermination exacte de la poussce hydraulique. Elle est fonction en effet : de la perte de charge dans les labyrinthes, de la repartition des pressions sur les ceintures de la roue, du fonctionnement du tuyau d'équilibrage et, pour certains types de turbines, de la répartition des vitesses à l'entrée de la roue. Ces différents facteurs sont en général peu accessibles au calcul, surtout pour les charges partielles de la turbine.

De mème, les cavitations à la sortie de la roue, les vibrations, ete., influencent la poussée hy. draulique et peuvent la rendre instable.

La mesure de la poussée hydraulique est done intéressante à plusieurs points de vue. Elle permet de connaître exactement la charge supportée par les pivoteries et constitue une auscultation du fonctionnement de la turbine, l'usure des labyrinthes entrainant une augmentation de la poussée.

Dans les installations à axe vertical, l'appareil de butée repose sur une poutre, appelée pont ou croisillon, suivant sa construction. Bien que cette poutre soit, en général, largement dimensionnée, elle se déforme sous l'effet des charges qui lui sont appliquées.

Une méthode simple pour la mesure de la poussće consiste à utiliser le dynamomètre constitué par cette poutre. L'étalonnage de ce dynamomètre s'obtient en mesurant la déformation due à une charge bien déterminée, le poids des parties tournantes par exemple. Les déformations du croisillon étant linéaires, ce qu'on peut d'ailleurs vérifier facilement, il est donc possible de mesurer la poussée et sa variation avec la charge de la turbine.

La seule difficulté de cette méthode se trouve dans la mesure des déformations qui sont faibles. Nous ayons obtemu de bons résultats en utilisant le niveau à bulle de M. l'Abbé Cayère (1).

Les caractéristiques du niveau que nous avons utilisé pour nos essais sont les suivantes :

Rayon de la bulle........... 53 mètres Nombre de divisions de la bulle. . 120 Grossissement du microscope... 30 environ

Une dénivellation de 1 micron entre deux points situés à un mètre de distance provoque un déplacement de la bulle de 1,59 division. Il nous était donc possible de mesurer avec cet appareil des pentes inférieures à $0,5 \cdot 10^{-6}$ radian.

Les déformations sous la poussée hydraulique maximum atteignaient quelque $0,1 \mathrm{~mm}$ par $\mathrm{m}$ correspondant à des pentes de quelques $10^{-4}$ radian.

La précision de l'appareil, qui peut d'ailleurs ètre doublée en utilisant une bulle de 100 mètres de rayon, est donc amplement suffisante.

La méthode fort simple consiste à fixer le niveau sur le croisillon et à observer les déplacements de la bulle.

(1) Pour la description de cet appareil voir l'article cití dans la bibliographie. 


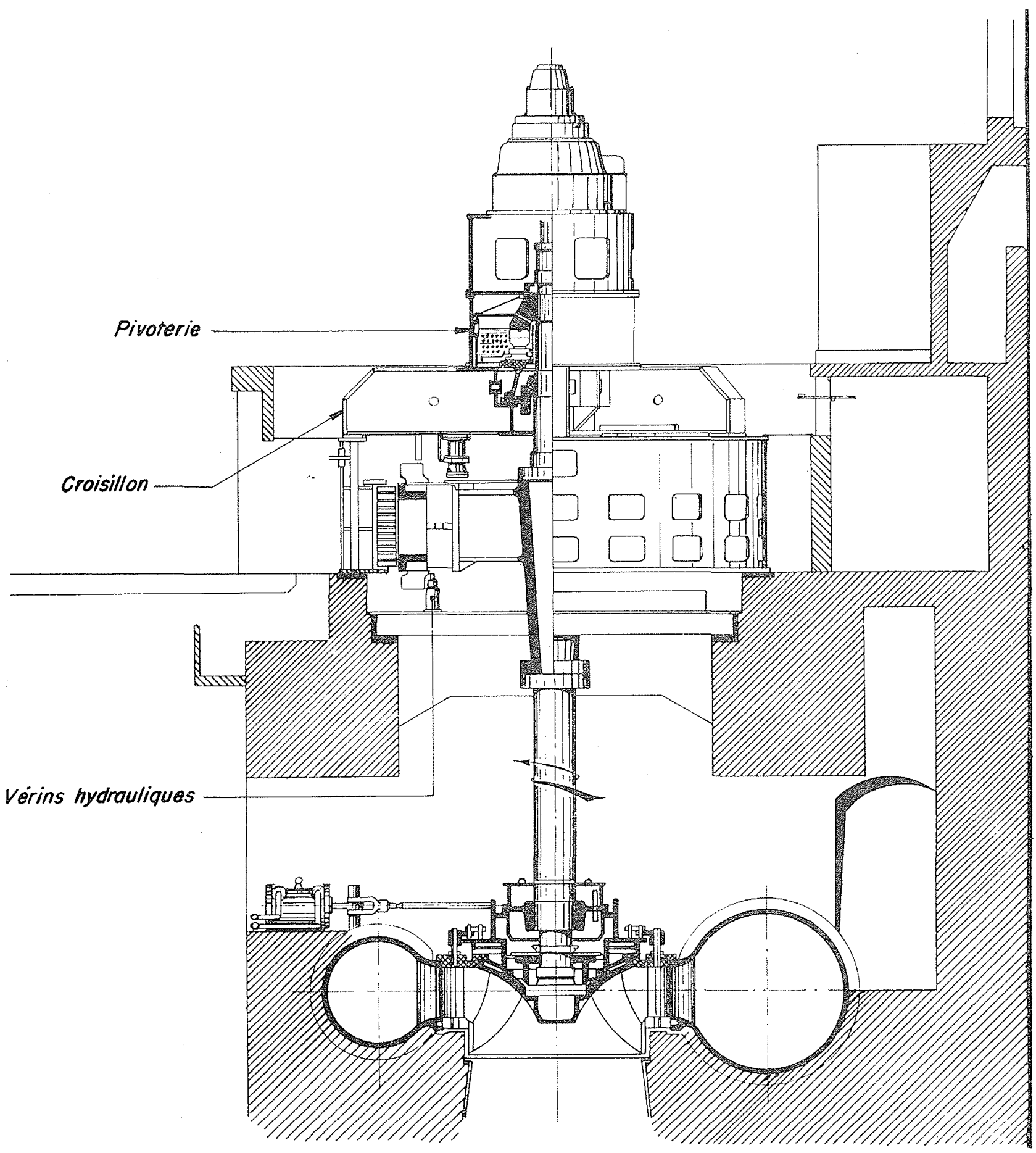

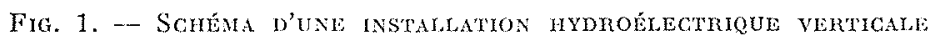

La figure 1 représente schématiquement un groupe hydroélectrique à axe vertical. La pivoterie repose sur le croisillon qui s'appuie lui-même sur le stator de l'alternateur. Des vérins hydrauliques permettent de soulever les parties tournantes.

Le groupe reposant sur ces vérins, le croisillon ne supporte aucune charge, on fixe alors le niveau en un point convenable puis on regle la position de la bulle. On laisse ensuite reposer le groupe sur le croisilion et l'on note la déviation de la bulle. Celte opération constitue l'étalonnage et permet de déterminer quelle est la charge qui correspond à une division de la bulle. 
La mesure de la pousséc hydraulique et de sa variation avec la charge est ensuite chose facile. Il faut toutelois gue les vibrations du groupr. soient négligeables.

On caicule, en senéral, la poussée à l'aide de la formule semi-empirique suivante :

$$
\mathrm{P}=l_{i} \cdot \frac{\pi \mathrm{D}^{2} s}{4} \cdot \mathrm{H}=k \cdot \mathrm{S} \cdot \mathrm{H}
$$

dans laquelle :

$P$ représente la charge en tonnes,

$H$ la hauteur de chute en mètres,

Ds le diametre de sortic de la roue en metres et partant,

$S$ la section de sortie en $\mathrm{m}^{2}$.

Le coefficient $k$, qui se rapporte à la poussée maximum, dépend du nombre de tour's spécifiques $N$ s de la turbine ainsi que de sa construction (forme des labyrinthes).

Nous avons exécuté maints essais sur des turbines de différents $\mathrm{Ns}$ pour déterminer $k$. Nous ne donnerons ici que les résultats d'expériences effectuées sur des turbines Francis de Ns 100, 200 et 400 .

\section{FRANCIS VERTICALE Ns 100}

a) Calcul de la poussée.

Pour ce Ns, la forme de la roue est schématiquement représentée par la figure 2.

La poussée dynamique est dans ce cas facile à calculer car la vitesse à l'entrée $C_{1}$ n'a pas de composante axiale.

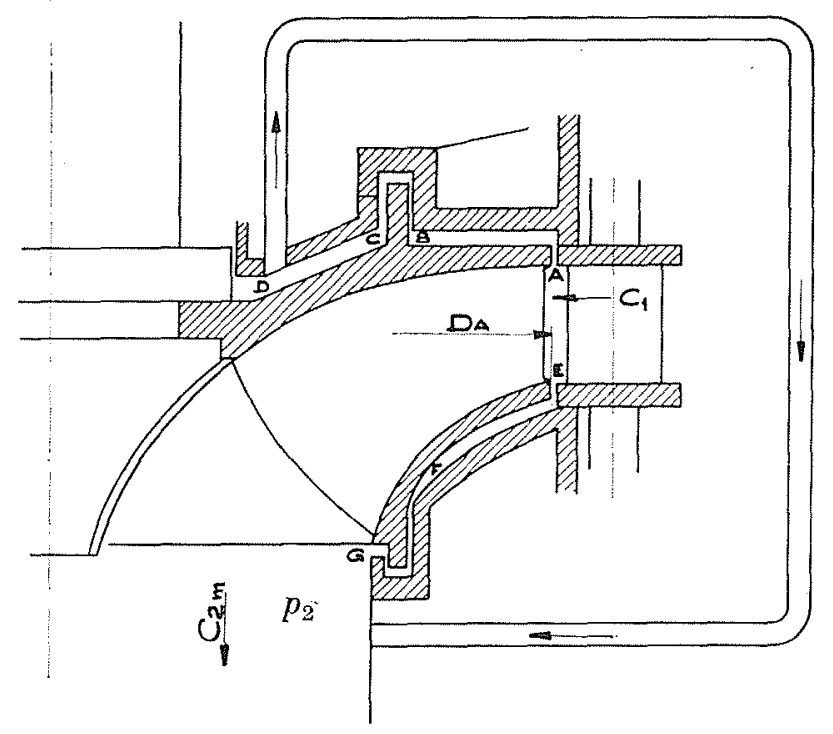

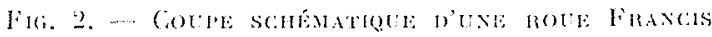
DE NS 100
Celle poussée, qui lend à décharger la pivolerie, est égale à la projection sur l'axe de la variation de quantité de mouvement, soit:

$$
\mathrm{P}=q \cdot \mathrm{S} \cdot \mathrm{C}_{2}^{2} \mathrm{~m}
$$

La répartition des pressions sur les ceintures de la roue est par contre difficile à déterminer par le ealcul. Examinons la répartition sur la ceinture supérieure. Si la hauteur de la chambre $A B$ est faible, par rapport à sa largeur, on peut assimiler l'écoulement dans cette chambre à celui provoqué par un disque tomrnant dans un carter étroit. L'écoulement radial se produit alors dans les couches limites, le long du disque et du carter, tandis que l'anneau central $a b c d$ (ng. 3 )

Frg. 3. - Ecoulentent Provogué par UN DISQUe TOURNANT DAXS UX CAIRTEH ExTor't

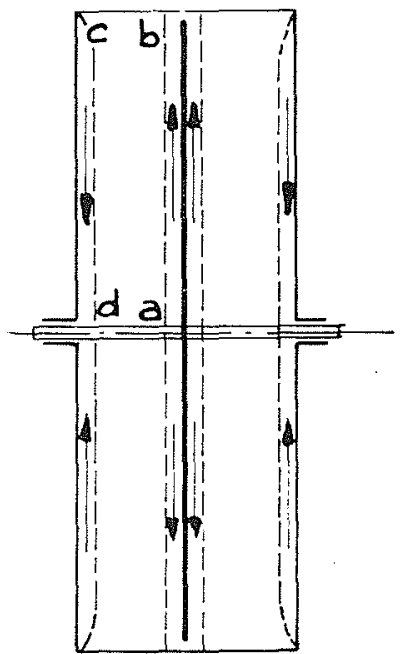

tourne en bloc comme un corps solide. La vitesse de rotation $\omega^{\prime}$ de cet anneau liquide est voisine de la moitié de celle du disque w. On pourra consulter à ce sujet les études expérimentales et thériques de F. Schuxz Gronow.

On a donc:

$$
\frac{1}{p} \cdot \frac{d p}{d r}=(1)^{\prime 2}=\frac{\omega^{2}}{4} r
$$

d'où :

$$
\frac{P}{\gamma}-\frac{P_{A}}{\gamma}=\frac{1}{g} \frac{\omega^{2}}{32}\left(\mathrm{D}^{2}-\mathrm{D}_{\mathrm{A}^{2}}\right)
$$

avec :

$$
\frac{p_{A}}{\gamma_{i}}=\mathrm{H} \cdots \frac{\mathrm{C}_{1}{ }^{*}}{2 g}-\varphi
$$

$H=:$ difrérence de cote entre le point $A$ el le plan d'eau amont;

$\%=$ pertes de charge. 
Remarquons toutefois que nous avons négligo I'influence de l'écoulement axial dù à la fuite au labyrinthe ot que, partant, l'équation (3) n'est pas rigoureusement exacte.

I a chute de pression $H_{1}$ dans le labyrinthe est d'autre part liée au débit de fuite, c'est-à-dire au débit du tuyau d'équilibrage; on a la relation :

$$
\begin{aligned}
\mathrm{H}_{1}=l \cdot\left(\frac{\mathrm{L}}{e}\right) \cdot \frac{\nu^{2}}{2 g} & +0,5 \frac{v^{2}}{2 g}+\frac{v^{2}}{2 g} \\
= & \left(f \frac{\mathrm{L}}{e}+1,5 \frac{v^{2}}{2 g}\right)
\end{aligned}
$$

dans laquelle :

$L=$ longueur du labyrinthe,

$e=$ jeu diamétral,

$0,5 \frac{y^{2}}{2 g},-\frac{v^{2}}{2 g}=$ pertes singulieres à l'entrie et à la sortie du labyrinthe,

$f=$ coefficient de frottement sans dimension.

Les parois dụ joint labyrinthe sont usinces soigneusement, on peut donc les considérel comme lisses. Dans ce cas, on a, d'aprés plusieurs expérimentateurs :

avec :

$$
f=0,316 \cdot R^{-\frac{1}{t}}
$$

$$
\mathrm{R}=\frac{\mathrm{De}}{\mathrm{y}}
$$

La relation (5) pent se mettre sous la forme :

avec :

$$
\text { Q fuite }=\alpha . \mathrm{S} \cdot \sqrt{2 g \cdot \mathrm{H}_{1}}
$$

$$
\alpha \text { coefficient de débil }=\frac{1}{\sqrt{f \frac{L}{e}+1,5}}
$$

$S=$ section de l'orifice annulaire.

La chute de pression dans le labyrinthe suppose donc la connaissance du débit de fuite $Q$ f. Nous avons admis pour le calcul de Q/ que les parois fixe et mobile du joint labyrinthe sont concentriques. Ce fait est en général inexact, nous rerrons en effet qu'aux charges partielles de la turbine, il existe une poussée hydraulique radiale, de direction fixe, qui tend à excentrer les labyrinthes. Cette excentricité augmente le débit de fuite.

Après le labyrinthe on peut, arec les mèmes hypothèses, supposer que la répatition des pres. sions daus la chambre CD est donnée par :

$$
\left.\frac{P_{0}}{\gamma}-\frac{P}{\gamma}=\frac{1}{9} \frac{\omega^{2}}{32} \cdot(\mathrm{D})^{2}-\mathrm{D}^{2}\right)
$$

avee:

$$
\frac{p_{\mathrm{B}}}{\gamma}-\frac{p_{\mathrm{O}}}{\gamma}=\mathrm{H}_{1}
$$

En négligeant la perte de charge dans le tuyau d'équilibrage (souvent l'écoulement dans ce tuyau n'est pas un écoulement en charge) on peut supposer $p_{\mathrm{L}}=p_{2}$.

Il est possible de calculer semblablement la répartition des pressions sur la ceinture inférieur. D'ailleurs, pour equilibrer la roue, on réunit quelquefois les deux chambres $A B$ et $E F$ par une tuyauterie.

Connaissant la répartition des pressions sur les ceintures supérieure et inférieure et la pression à la sortie de la roue $p_{2 .}$, on peut calculer la poussée totale sur la roue. Les calculs sont laborieux et peu précis. Par contre, la méthode d'essais décrite permet de déterminer simplement la poussée, ainsi que sa variation avec la charge de la turbine.

\section{b) Résultats dessais :}

Pour le Ns considéré, nous avons effectuć des mesures sur deux turbines identiques dont les caractéristiques sont les suivantes (centrale souterraine de Brommat) :

$$
\begin{aligned}
\mathrm{N} & =42.500 \mathrm{ch} . \\
n & =500 \mathrm{t} / \mathrm{min} . \\
\mathrm{H} & =255 \mathrm{~m} ; \text { lors des essais } 253 \mathrm{~m} . \\
\mathrm{DS} & =1.275 \mathrm{~mm} .
\end{aligned}
$$

Les résultats sont représentés sur la figure 4 où nous ayons porté en abscisses les charges, et en ordonnées les poussées en tonnes.

La poussée maximum, qui se produit à pleine charge, atteint 49,5 tonnes, tandis cue la poussée à vide n'est que de 18 tonnes.

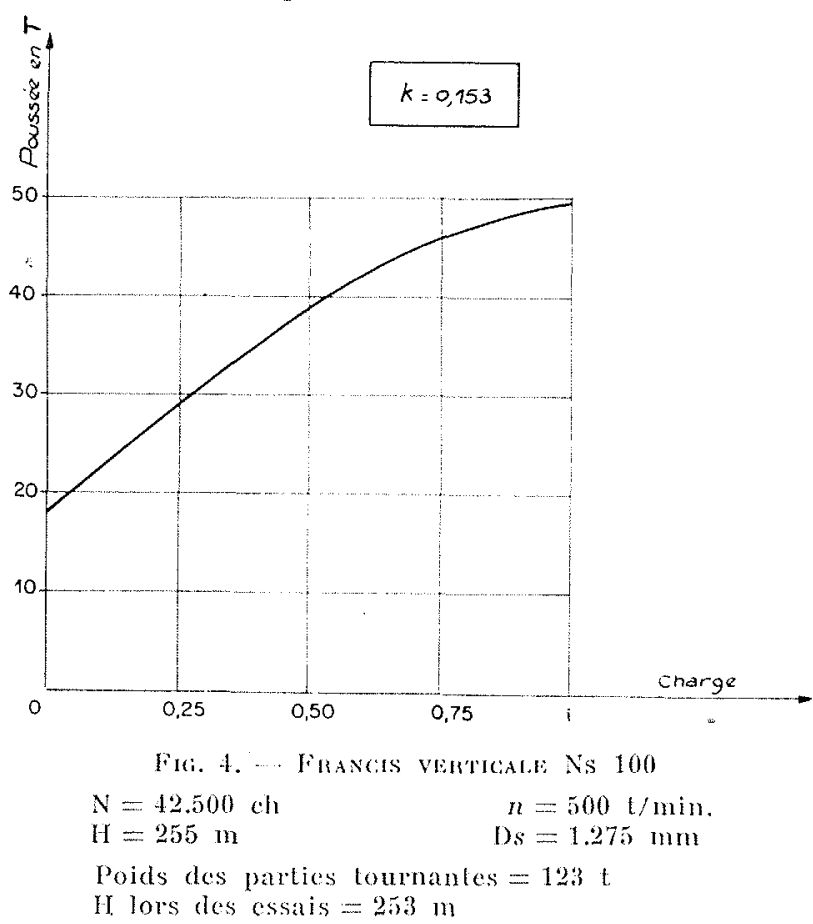


Les incidents de pivoterie se produisant, en général, pendant les périodes de démarrage et d'arrêt, il est intéressant de connaître les poussées à vide.

On a donc, pour un Ns 100 :

$$
\begin{aligned}
& P_{\text {max. }}=0,153 \mathrm{~S} . \mathrm{H} . \\
& \mathrm{P}_{\text {vide }}=0,056 \mathrm{~S} . \mathrm{H} .
\end{aligned}
$$

Le rapport de la poussée maximum au poids des parties tournantes est, d'autre part, 0,4.

\section{FRANCIS VERTICALE Ns 200}

Les mesures ont été effectuées sur une turbine dont les caractéristiques sont (centrale de Cordéac) :

$$
\begin{aligned}
\mathrm{N} & =56.000 \mathrm{ch} . \\
n & =214 \mathrm{t} / \mathrm{min} . \\
\mathrm{H} & =89 \mathrm{~m} ; \text { lors des essais } 86,5 \mathrm{~m} . \\
\mathrm{Ds} & =2.750 \mathrm{~mm} .
\end{aligned}
$$

Les résultats d'essais sont donnés par la figure 5 .

Les poussées maximum et à vide atteignent respectivement 130 et 40 tonnes, soit :

$$
\begin{aligned}
& \mathrm{P}_{\text {max. }}=0,253 \mathrm{~S} . \mathrm{H} . \\
& \mathrm{P}_{\text {vide }}=0,078 \text { S.H. }
\end{aligned}
$$

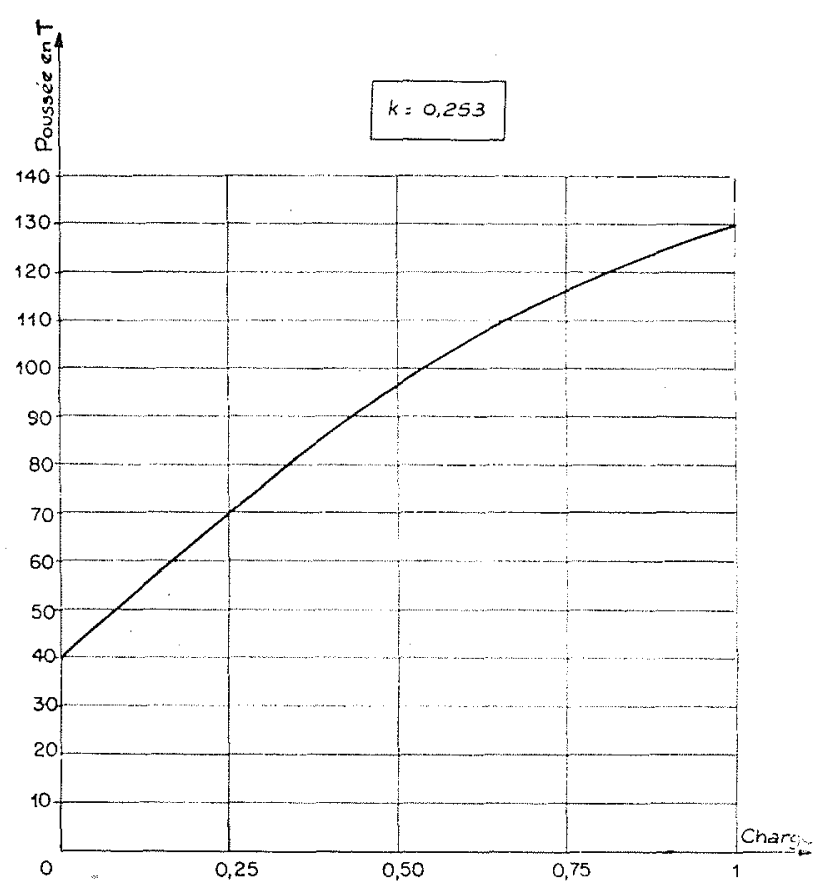

Fic. 5. - Francrs venticale Ns 200

$\mathrm{N}=56.000 \mathrm{ch}$

$\mathrm{H}=89 \mathrm{~m}$

$n=214 \mathrm{t} / \mathrm{min}$

Poids des parties tournantes $=230 \mathrm{t}$

H lor's des essais $=86,5 \mathrm{~m}$
Le rapport de la poussée max. au poids des parties tournantes est, d'autre part, 0,565 .

\section{FRANCIS VERTICALE Ns 400}

Les mesures ont été faites sur une turbine dont les caractéristiques sont (centrale du Drac inférieur) :

$$
\begin{aligned}
N & =2.660 \mathrm{ch} . \\
n & =250 \mathrm{Hin} . \\
H & =16,5 \mathrm{~m} . \\
\mathrm{Ds} & =1.700 \mathrm{~mm} .
\end{aligned}
$$

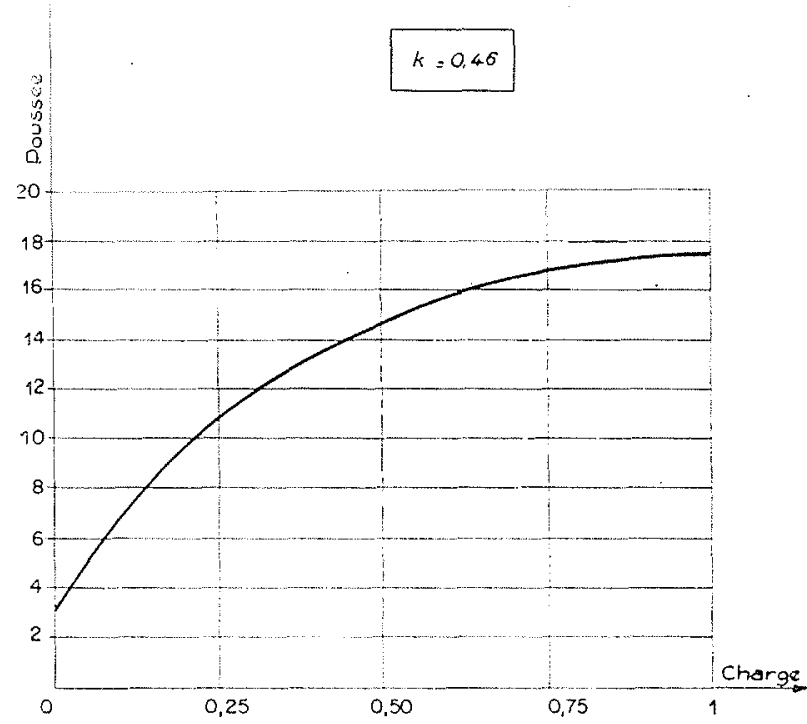

Fig. 6. - Francis verticale Ns 400

$$
\begin{aligned}
& \mathrm{N}=2.660 \mathrm{ch} \\
& \mathrm{H}=16,5 \mathrm{~m}=250 \mathrm{t} / \mathrm{min} . \\
& \text { Poids des partics tournantes }=17 \mathrm{t}
\end{aligned}
$$

La figure 6 donne les résultats d'essais.

Les poussées maximum et à vide atteignent respectivement 17,5 et 3 tonnes.

Elles correspondent aux formules :

$$
\begin{aligned}
& P_{\text {max. }}=0,46 \text { S.H. } \\
& P_{\text {vithe }}=0,080 \text { S.H. }
\end{aligned}
$$

Le poids des parties tournantes est, à très peu près, égal à la poussée hydraulique maximum.

Pour cette turbine, les mèmes essais ont été repris par la division «Technique générale»de la «Production hydraulique»(EDF). Les déformations du croisillon étaient mesurées à l'aide 
d'extensomètre à fil résistant. Les résultats obtenus par cette dernière méthode sont en fort bon accord avec les nôtres.

\section{CONCLUSION}

Les différents essais effectués nous ont permis d'établir une formule empirique valable pour des turbines Francis, de construction bien définic.

La figure 7 donne les valeurs du coeficient $l$, de la formule $\mathrm{P}=k$.S.H., pour la poussée maximum et la poussée à vide.

Nous avons signalé qu'il existe, dans les lurbines hydrauligues, des poussée radiales dues à une alimentation et, par conséquent, à une ré-

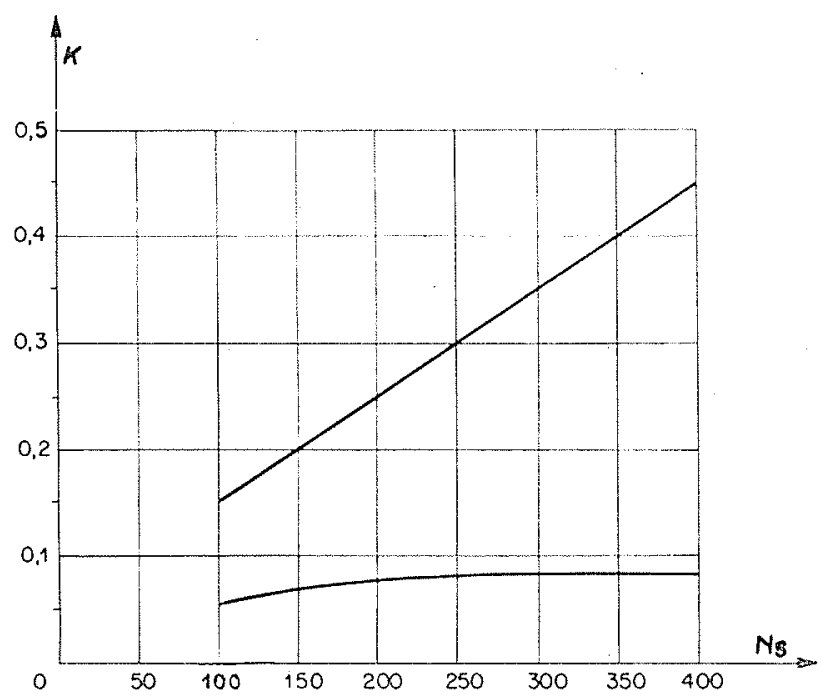

Fra. 7. - Grarhious donmant les valears du coefficient lí

$$
\mathrm{P}=\mathrm{k} \frac{\pi}{4} \mathrm{D}_{\mathrm{s}}^{2} \mathrm{H}
$$

en fonction du NS pour des turbines Francis.

partition des pressions à l'entrée de la roue, non uniformes. Cette poussée radiale a été mesurée pour des pompes centrifuges. A.J. STEPANoFF, dans son ouvrage : Gentrifugal and axial flow plumps, donne des résultats de mesures pour les poussées axiales et radiales des pompes centrifuges.

La poussce radiale des machines hydrauliques est négligeable pour la charge normale, c'est-àdire pour le rendement maximum. En effet, dans le cas des turbines hydraulicques, pour ce point de fonctionmement, l'alimentation est meilleure qu'à toute antre charge et la répartition des pressions est quasi uniforme à l'entrée de la roue.

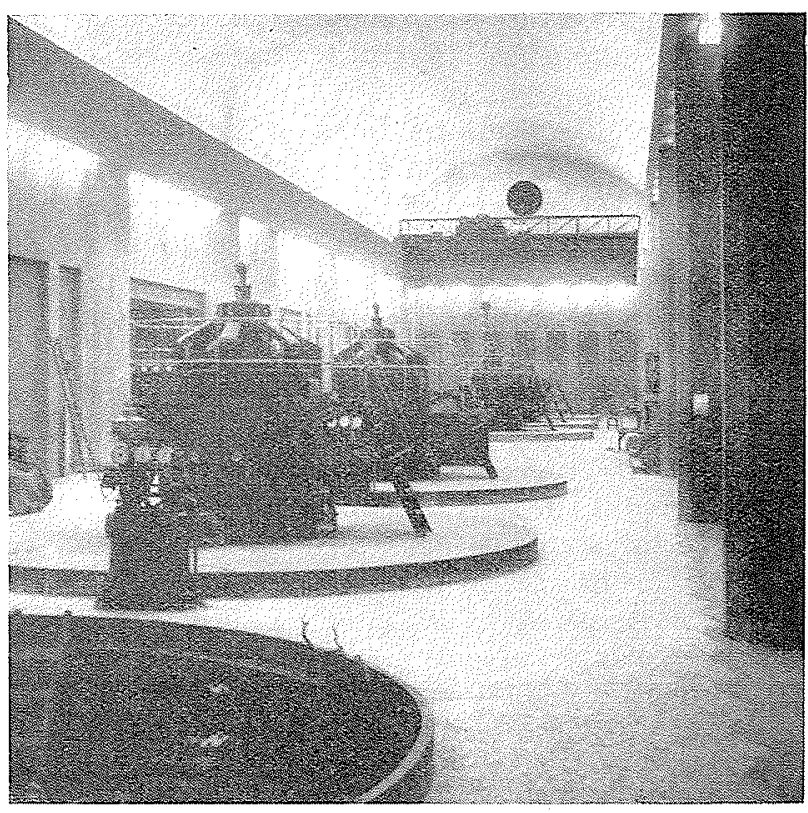

Fig. 8. Contrate de Brommat.

La direction de cette poussée est fixe, mais son intensité varie avec la charge de la turbine.

Dans certaines installations à axe horizontal, la pcussée radiale peut être importante aux charges partielles et provoquer un excentrement des labyrinthes. Si leur jeu radial est faible, il peut en résulter des vibrations analogues à celles des

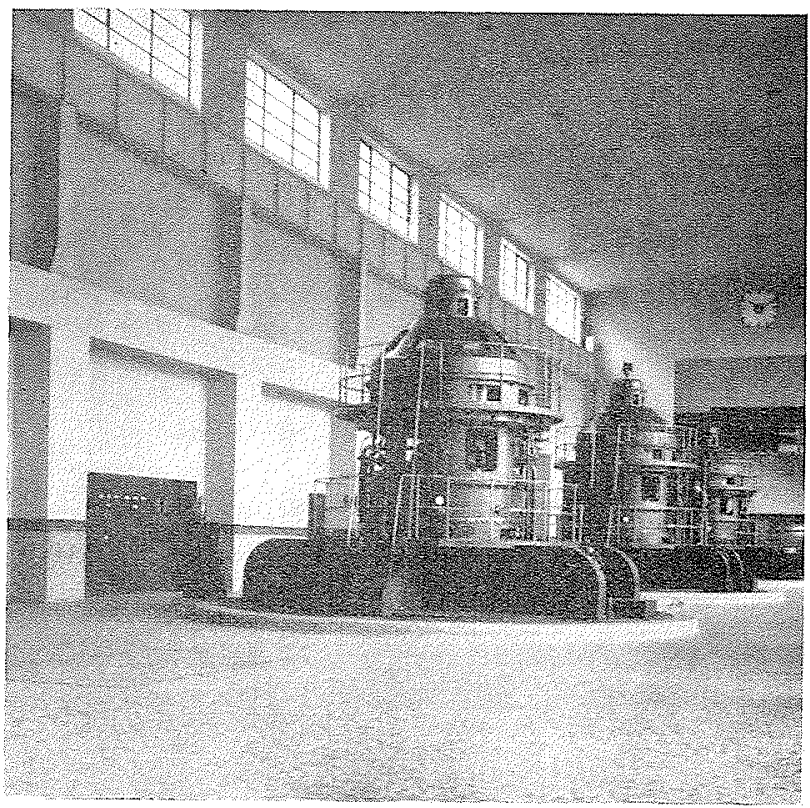

Fig. 9. - Centrille de Sarrays. 
paliers lisses. On arrive quelquefois à supprimer. les vibrations d'une turbine horizontale en excentrant légèrement les labyrinthes dans le sens convenable.

Pour les installations horizontales, la charge des butées se réduit à la poussée hydraulique. Il cst donc intéressant de la connaitre en intensité et direction. Nous avons mis au point un dispositif en permettant la mesure. Ce dispositif pourra d'ailleurs servir à contrôler l'usure des labyrinthes.

Remercions, en terminant, les services de l'E.D.F., ainsi que les industriels qui nous ont permis d'effectuer des mesures sur leurs installations. Nous devons signaler d'autre part que dans certaines centrales (Sarrans), où il a élé impossible de se protéger des courants d'air froid, ces mesures n'ont pu être effectuées avec précision car le niveau CaYère est très sensible aux variations de température.

Il eût été possible d'effectuer des mesures prócises dans cette centrale si les opérateurs avaient eu suffisamment de temps devant eux.

\section{BIBLIOGRAPHIE}

I. H. POLHEMUS and J. HENLY. Dredge pumps and thrust loads. Trans. A.S.M.E., vol. 51, de 1929 .

STEPANOFF.

Leakage loss and Axial thrust in centrifugal pumps. Trans. A.S.M.E., vol. 54 , de 1932.

F. SCHULZ-GRUNON.

Der Reibungwiderstand rotiererder Scheiben in Gehäusen.

Z. für Angewandte Matematik und Mekanik, juillet 1935.

STLPANOFF.

Centrifugal and axial flow pumps.

New-York, John Wiley and Sons (1948).

Abbé CAYERE, Ing. A. et M. et I.E.G., Directeul de l'Ecole Libre d'Apprentissage de Grenoble.

L'emploi du niveau à bulle en métrologie.

Mesures et contróle industriel, n ${ }^{0} 134$, juillet 1948.

Testing Topies, Jan., Feb., Mar. 1949.

Strain Gages Help solve a difficult turbine thrust loading problem at Baldwin.

Dans ce dernicr artiele, l'auteur décrit un dispositî́ d'essais permettant de déterminer la poussée axiale des modèles reduits de Turbines Hydranliques.

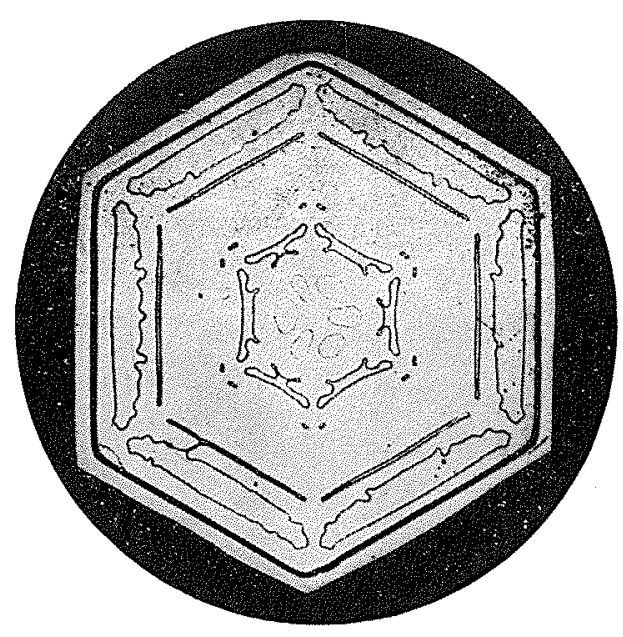

